\title{
Comportement d'une turbomachine dans un circuil hydraulique soumis à des fluctuations de pression et de débit
}

\author{
Behaviour of a turbomachine in a hydraulic system \\ subject to fluctuating pressure and flow
}

Pierre Boussuges et Dimitry Khaletzky

Société Neyrpic - Grenoble

\section{Définition du problème}

\section{Régimes transitoires}

Tout circuit hydraulique est destiné à subir un certain nombre de régimes transitoires. Ces régimes s'accompagnent d'ondes de choc se propageant dans tout le circuit et pouvant provoquer localement de fortes variations de pression ou de débit. Il est important de prévoir ces variations, ce qui implique la connaissance assez précise du comportement de chaque composant du circuit en régime transitoire.

Diverses méthodes permettent de décrire ce comportement. Elles sont en général dérivées de la méthode graphique de Bergeron : on considère l'onde se déplaçant le long du circuit. Toutes les fois qu'elle traverse un composant donné (vanne, conduite, turbomachine), le débit et la pression sont obtenus à l'aide des courbes caractéristiques $H(Q)$ de l'appareil en question. Ces courbes caractéristiques sont obtenues expérimentalement en régime permanent. Ainsi, le régime transitoire est décrit par une succession de régimes permanents.

Ce type de calcul fournit, en bon accord avec la réalité, les lois de manœuvre usuelles d'un circuit hydraulique, même complexe (arrêt, démarrage, déclenchement, emballement). Les programmes de régulation sont fondés sur le même principe.

Ces méthodes sont valables tant que les transitoires décrits sont lents, ou en d'autres termes, tant que les caractéristiques des régimes permanents ne sont pas trop distordues.

\section{Résonance hydraulique}

Si un composant du circuit génère des ondes de choc rapprochées dans le temps, nous sommes en présence d'un phénomène vibratoire ou transitoire rapide. Les ondes successives provoquent des fluctuations de débit et de pression, dont nous noterons $\omega$ la pulsation. Dans les équations du mouvement et de la continuité, il apparait un terme instationnaire proportionnel à $\omega$.

Si ce terme n'est plus négligeable, l'hypothèse faite au paragraphe précédent n'est plus valable et les méthodes classiques risquent de donner des résultats erronés.

Le but final de l'étude entreprise est la mise au point d'une méthode décrivant les phénomènes vibratoires dans les circuits hydrauliques complexes, comprenant en particulier une ou plusieurs turbomachines. Cette méthode n'utilisera pas les données expérimentales obtenues en régime permanent, mais devra être fondée sur les équations instationnaires du mouvement et de la continuité d'un fluide réel. Elle permettra d'étudier le comportement du circuit dans son ensemble, et notamment ses fréquences propres, c'est-à-dire celles pour lesquelles il risque d'entrer en résonance.

Pour un composant autre qu'une turbomachine, la résolution numérique des équations instationnaires complètes de l'hydrodynamique est envisageable. Mais ce n'est pas le cas pour une turbomachine, où l'écoulement est tridimensionnel, de géométrie complexe et où les pièces susceptibles de vibrer sont nombreuses.

Devant l'impossibilité de résoudre ce problème dans son ensemble, nous sommes amenés à considérer un schéma de machine très simplifié.

Fanelli $[1,2]$ considère les aubages comme une cascade de plaques planes placées dans un écoulement et dont l'angle d'incidence par rapport à celui-ci varie. Cette approche intéressante ne tient pas compte cependant de l'inertie du fluide en rotation dans la roue. Le point de départ de notre démarche consiste au con. traire à décrire le mouvement instationnaire des veines 


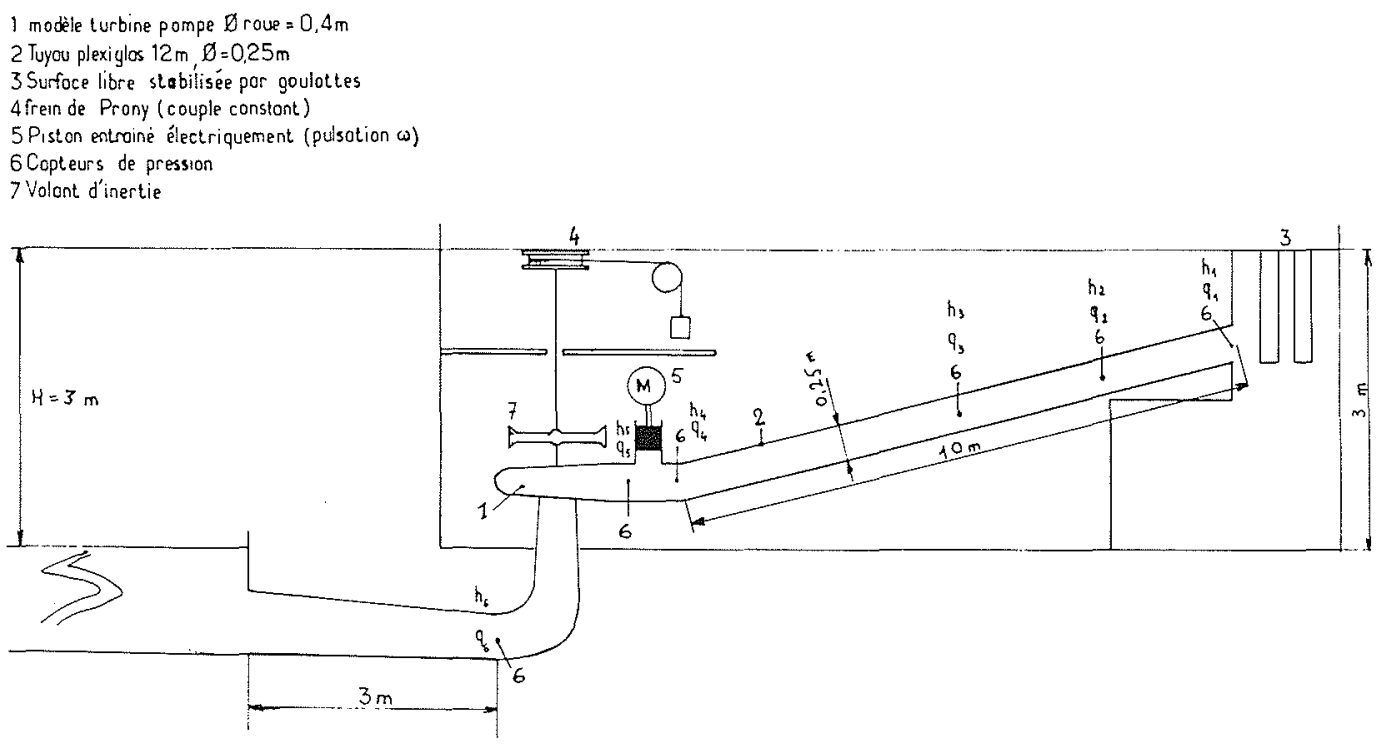

Figure 1. - Schéma du dispositif expérimental.

liquides circulant à l'intérieur de la machine. Les équations hydrauliques monodimensionnelles appliquées aux turbomachines sont traitées par la méthode des impédances [3].

\section{Principes de l'étude}

Nous étudions le comportement d'un circuit hydraulique simple fonctionnant en régime stationnaire oscillant. Ce circuit, représenté sur la figure 1 , se compose d'un réservoir amont, une conduite, une dérivation équipée d'un piston, un modèle de turbine-pompe, un aspirateur, un réservoir aval. $\mathrm{La}$ machine fonctionnant en turbine, est freinée par un frein de Prony créant un couple résistant constant.

\section{Partie théorique}

La turbomachine hélico centrifuge est considérée comme $N_{d}$ conduites parallèles identiques fixes prolongées sans discontinuité par $N_{r}$ conduites mobiles.

$N_{d}$ est le nombre de directrices, $N_{r}$ est le nombre d'aubages de la roue.

Si $\Delta q$ est la fluctuation de débit à la sortie d'un canal du distributeur, la fluctuation à l'entrée d'un canal de la roue est $\left(N_{d} / N_{r}\right) \times \Delta q$.

Ce modele ne tient pas compte des fluctuations provoquées par le défilement des grilles fixes devant les grilles mobiles, pas plus qu'il ne décrit les éventuels phénomènes de torche. Cependant le but de l'étude n'est pas la détermination théorique du spectre émis par une turbomachine. Il s'agit au contraire de déterminer son comportement passif vis-à-vis d'une perturbation sinusoïdale externe. Ce comportement se traduit par un nombre complexe, fonction de la fréquence des oscillations que l'on nommera impédance de la machine $Z=\Delta h / \Delta q$ où $\Delta h$ est la différence de fluctuation de chute entre l'entrée et la sortie de la machine, et $\Delta q$ est cette fois la fluctuation totale de débit.
Cette impédance, associée à celles des autres composants du circuit permet de déterminer les fréquences critiques de celui-ci. Alors, si ces fréquences correspondent aux fréquences émises par la machine (lesquelles sont déterminées par ailleurs sur plate-forme d'essais) il y a lieu de conclure à un risque de résonance hydraulique.

\section{Méthode des impédances - Application d'une conduite rectiligne}

Cette méthode, inspirée de l'étude des circuits électriques, a été particulièrement développée par Streeter [3]. Elle est particulièrement adaptée à l'étude d'un régime stationnaire oscillant dans une conduite, à la condition que l'amplitude des oscillations soit faible devant les grandeurs moyennes.

Considérons une conduite rectiligne de longueur $L$. Les équations du mouvement et de la continuité d'un fluide traversant cette conduite sont :

$$
\begin{aligned}
& \frac{\partial H}{\partial x}+\frac{1}{g A} \frac{\partial Q}{\partial t}+\frac{f Q^{2}}{2 g D A^{2}}=0 \\
& \frac{\partial Q}{\partial x}+\frac{g A}{a 2} \frac{\partial H}{\partial t}=0
\end{aligned}
$$

$H$ est la pression exprimée en mètres d'eau $Q$ est le débit

$a$ est la célérité du son dans la conduite

$f=\Delta H / L V^{2}$ le coefficient de perte de charge

$A, D$ la section et le diamètre de la conduite

$\Delta H$ la perte de charge

$g$ l'accélération de la pesanteur

Décomposons la pression et le débit en partie moyenne et partie instantanée.

$$
\begin{aligned}
& H=\bar{H}+h e^{i \omega t} \\
& Q=\bar{Q}+q e^{i \omega t}
\end{aligned}
$$

$\omega$ est la pulsation de la perturbation et $i=\sqrt{-1}$. 
Introduisons ces expressions dans les équations (1) et (2).

Si $|h| / \bar{H} \ll 1$ et $|q| / \bar{Q} \ll 1$, le terme de frottement de l'équation (1) peut être linéarisé et l'on obtient un système différentiel aux conditions initiales dont les inconnues sont $h$ et $q$.

$$
\begin{aligned}
& \frac{\partial h}{\partial x}+\left[R+\frac{i \omega}{g A}\right] \quad q=0 \\
& \frac{\partial q}{\partial x}+\frac{i g A \omega}{a 2} \frac{\partial h}{\partial x}=0 \\
& \text { où } R=(2 f \bar{Q}) /\left(2 g D A^{2}\right)
\end{aligned}
$$

Dans le cas d'une conduite à section constante, ce système s'intègre analytiquement et fournit deux relations linéaires entre le couple $(h, q)$ à l'entrée de la conduite et à sa sortie.

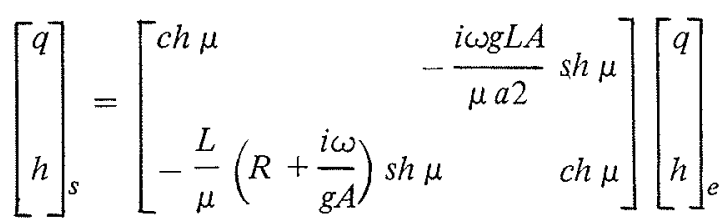

La matrice carrée qui apparait dans la relation (5) sera nommée par la suite "matrice de transfert" de la conduite.

\section{Application à la turbo-machine}

Les canaux du distributeur sont considérés comme des conduites à section variable et l'équation (3) est

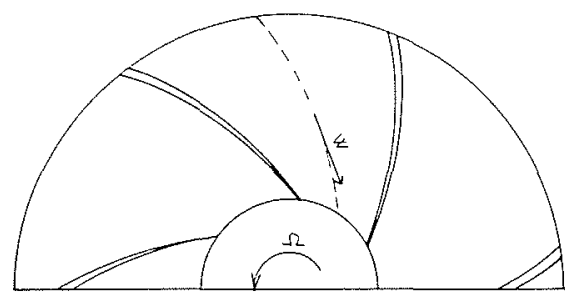

VUE EN PLAN DE LA ROUE
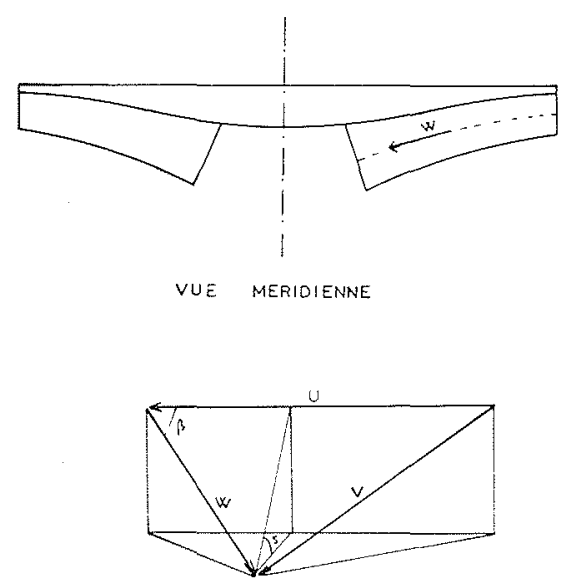

TRIANGLE DE VITESSES

Figure 2. intégrée numériquement suivant une ligne de courant. Etant donné la faible longueur de ces canaux, l'équation (4) se réduit à $\partial q / \partial x=0$, soit $q_{e}=q_{s}$ (fonctionnement sans cavitation).

Les canaux de la roue sont traités suivant le même principe ; mais l'équation (1) est compliquée par la rotation de la roue. De plus, la géométrie de l'écoulement nécessite plus d'attention.

La figure 2 schématise la roue de la machine. $U$ est la vitesse d'entrainement d'une particule fluide, $W$ sa vitesse relative par rapport à la roue, $V$ sa vitesse absolue.

Sous forme vectorielle $\vec{V}=\vec{U}+\vec{W}$ et en élevant au carré : $V^{2}=W^{2}-U^{2}+2 U . V_{u}$

$V_{u}$ est la projection de la vitesse absolue sur la vitesse d'entraînement. Le mouvement absolu du fluide dans un canal doit vérifier l'équation

$$
\frac{\partial \vec{V}}{\partial t}+\overrightarrow{\operatorname{grad}} \frac{V^{2}}{2}-\vec{V}_{\Lambda} \overrightarrow{\operatorname{rot}} V+g \overrightarrow{\operatorname{grad}} H=0
$$

Soient :

$$
H_{\mathrm{rel}}=H+\frac{W^{2}-U^{2}}{2 g}
$$

et $\beta$ l'angle des directions de $\vec{V}$ et $\vec{W}$.

L'équation (6) devient, en projection sur une ligne de courant relative :

$$
g \frac{\partial H r}{\partial \sigma}=\frac{\partial W}{\partial t}-\frac{\partial U}{\partial t} \cos \beta
$$

Dans cette relation $\sigma$ est l'abscisse curviligne d'une ligne de courant caractéristique. Des considérations trigonométriques permettent de déduire de la figure 2.c la relation :

$$
d \sigma=\frac{d r}{\sin \beta \cos \zeta}
$$

$r$ étant la distance de la particule considérée par rapport à l'axe de la roue.

Avant d'appliquer la méthode des impédances aux différentes grandeurs de l'équation (8), nous introduisons l'hypothèse supplémentaire : les angles $\beta$ et $\zeta$ ne fluctuent pas. Ainsi, $\partial \beta / \partial t=0$ et la relation (9) peut fournir une fois pour toutes $\sigma$ en fonction de $r$ ou l'inverse.

L'équation (8) devient:

$$
\frac{\partial h r}{\partial \sigma}+\frac{i \omega q}{g S}+r \cos \beta i \omega \theta=0
$$

$$
\text { avec } \begin{aligned}
& H r=\overline{H r}+h r^{e i \omega t} \\
& Q=\bar{Q}+q^{e i \omega t} \\
& \Omega=\bar{\Omega}+\theta^{e i \omega t}
\end{aligned}
$$

A l'interface distributeur-roue, il convient de différencier l'équation (7) pour passer de la fluctuation de 
pression "absolue" $h$, à la fluctuation "relative" $h r$. $g(h r-h)=\left(2 R^{2} \bar{\Omega}-\frac{\bar{Q}}{2 \pi e} \cos \beta\right) \theta-\frac{\Omega N r}{2 \pi e} q \cos \beta(1)$

Pour que le système d'équations soit complet, il faut une dernière relation définissant $\theta$ (fluctuation de vitesse angulaire).

Cette relation est obtenue par différenciation des deux relations suivantes:

$$
\begin{aligned}
& C_{m} \Omega=\eta \rho g Q\left(H_{e}-H_{s}\right)+\frac{\partial E c}{\partial t} \\
& I \frac{d \Omega}{d t}=C_{m}-C m o
\end{aligned}
$$

$C_{m}$ est le couple moteur

$I$ le moment d'inertie des parties tournantes

$\eta$ le rendement

$E_{c}$ l'énergie cinétique de l'eau dans les canaux de la roue

Le résultat est une équation linéaire du premier ordre en $\theta$, faisant intervenir he et $h s$. Cette nouvelle relation représente une difficulté numérique non négligeable, puisqu'elle transforme le système de "conditions initiales" $(3)+(11)+(10)$ en un problème "aux limites".

\section{Partie expérimentale}

Rappelons que le but de l'essai est d'obtenir la valeur du nombre complexe $Z(2 \pi f)=\left(h_{s}-h_{e}\right) / q$, la fréquence $f$ des perturbations variant de 0 à $15 \mathrm{~Hz}$.

Il est possible d'obtenir les spectres $h_{s}$ et $h_{e}$ sans problèmes majeurs. La fluctuation de débit $q$ demeure cependant difficilement accessible. Pour cette raison, nous avons voulu mettre au point une technique de mesure indirecte, permettant en théorie, d'éviter les mesures de $q$.

\section{Principe de la mesure indirecte}

Considérons l'installation de la figure 1. Supposons que l'on connaisse la matrice de transfert de la conduite,

(1) $e$ est la hauteur du canal du distributeur. ou simplement le rapport $m_{11} / m_{21}$, où $m_{i 1}$ sont les éléments de la première colonne de la matrice. Alors, un calcul très simple fournit

$$
Z=\frac{h_{6}-h_{5}}{m_{11} / m_{21} h_{4}+q_{0}}
$$

$q_{0}$ est le débit généré par le piston.

On voit que cette expression est exempte de toute fluctuation de débit mesurée.

\section{Essai actuel}

La technique de la mesure indirecte, malgré un côté attrayant, a l'inconvénient de supposer que la matrice de transfert de la conduite est proche de sa valeur théorique. Des essais préalables sur un circuit simplifié dans lequel la machine était remplacée par un injecteur de turbine Pelton ont montré que ce n'est pas toujours le cas. En conséquence, nous reprenons les essais en utilisant un débitmètre à ultrasons qui sera disposé entre le piston et la machine. Nous espérons que cette nouvelle installation permettra d'obtenir des valeurs satisfaisantes de l'impédance d'une turbomachine.

\section{Conclusion}

L'étude entreprise consiste à déterminer théoriquement et expérimentalement, l'impédance d'une turbomachine. La partie théorique, fondée sur la méthode des impédances, est achevée. En ce qui concerne la partie expérimentale, des essais sont en préparation au cours desquels nous effectuerons simultanément une mesure indirecte de fluctuations de débit à l'aide d'une conduite rectiligne de matrice de transfert connue, et une mesure directe, grâce à un débitmètre à ultrasons.

\section{Bibliographie}

[1] FANELLI M. - Further considerations on the dynamic behaviour of hydraulic turbomachinery. Water Power June 1972.

[2]FANELLI M. - Les phénomènes de résonance hydraulique La Houille Blanche, 1975.

[3] STREETER V.L., WYLIE E.B. - Hydraulic Transients. Mc Graw Hill Book Co. Inc. New-York N.Y. 1967. 


\section{Discussion}

Président : M.J. ChEvalIER

M. CHEVALIER. - Merci M. KHALETZKY, Merci M. BOUSSUGES.

Je sais que vous auriez préféré attendre, pour présenter vos travaux, de pouvoir étayer la théorie par une expérimentation suffisante. Je crois pourtant qu'il était nécessaire que ce mémoire soit présenté aujourd'hui. En effet, il est intéressant qu'on soit au courant des travaux de recherche en cours dans les domaines qui nous intéressent ; d'autre part, je suppose que ce travail aura de nombreux développements ultérieurs, et que ce sera une incitation, pour nous, à revenir vous entendre. Ils sont très intéressants.

La discussion est ouverte.

M. ROSSI. - Quelques remarques au sujet de la pulsation de la caractéristique hydraulique des turbines à charge partielle.

Les turbines FRANCIS et les pompes-turbines fonctionnant en turbine sont parfois en même temps origine et siège de perturbations. Nous nous réfèrons en particulier aux fonctionnements à charge partielle. Les conséquences que ces perturbations entraînent très souvent sur le fonctionnement de tout le système justifient de nous entretenir sur le schéma d'interprétation de leur origine à l'intérieur de la machine.

Dans ces conditions, en effet, une intense rotation globale de la masse d'eau se produit dans le tuyau d'aspiration autour d'un axe qui, à son tour, tourne dans le même sens que la machine à une vitesse réduite à une fraction de la vitesse de rotation de la machine. Il en résulte le phénomène bien connu de la torche à l'aval.

La première conséquence est l'apparition dans le tuyau d'aspiration d'une onde de pression qui tourne à la même vitesse réduite. L'amplitude de cette pulsation de pression, relevée par un capteur placé tout prés, au-dessous de la roue, peut atteindre et dépasser des valeurs correspondant à $5 \%$ de la chute. Si l'aspirateur était rectiligne, cette pulsation serait la seule conséquence des phénomènes en question, puisque, à une certaine distance à l'amont et à l'aval, les pressions seraient pratiquement stabilisées ; mais la présence du coude d'aspiration, c'est-à-dire d'un élément asymétrique, fait que la rotation de la torche produit une altération périodique des performances instantanées de la machine.

HYDROART a effectué des essais préliminaires pour expliquer le phénomène et a trouvé que :

a) Tout près et en-dessous de la roue (B, fig. 1), la pulsation tournante est mise en évidence par l'enregistrement simultané des signaux de trois capteurs décalés de $120^{\circ}$ l'un par rapport à l'autre : les signaux sont également déphasés du même angle (fig. 2).

b) A une certaine distance à l'amont et à l'aval (A, C, fig. 1), les pressions relevées (toujours en trois points à $120^{\circ}$ ) pulsent

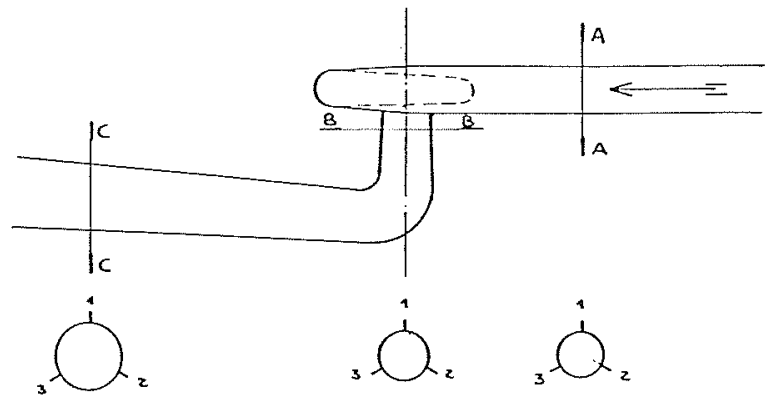

Figure 1. - Position des capteurs de pression sur la turbine modèle. en phase entre elles, avec une amplitude généralement inférieure à celle qu'on relève tout près et au-dessous de la roue et à la même fréquence (fig. 3,4 ).

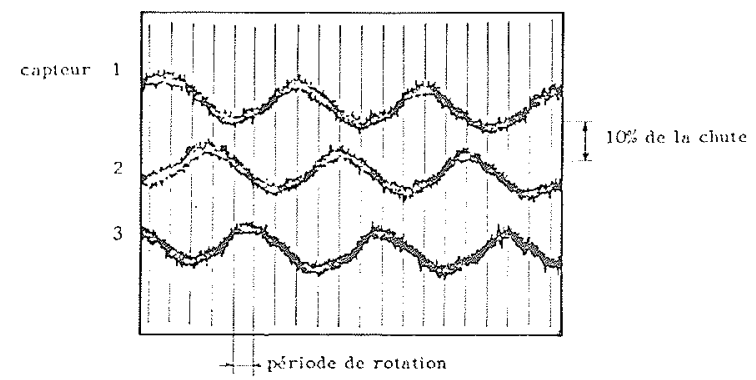

Figure 2. - Pulsation de la pression dans la section B (Les trois signaux sont déphasés de $120^{\circ}$ ).

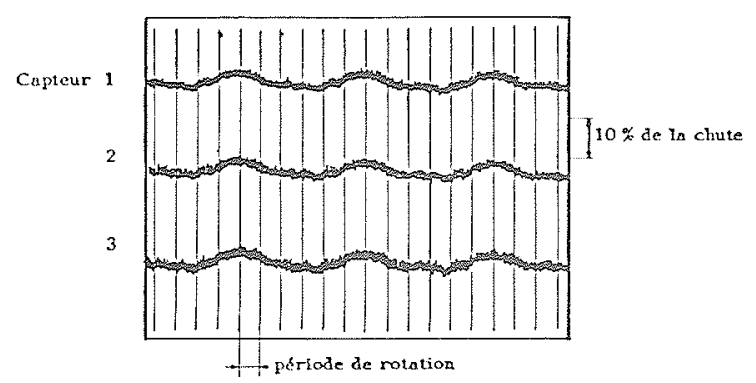

Figure 3. - Pulsation de la pression dans la section $A_{\perp}$ (Les trois signaux sont en phase entre eux).

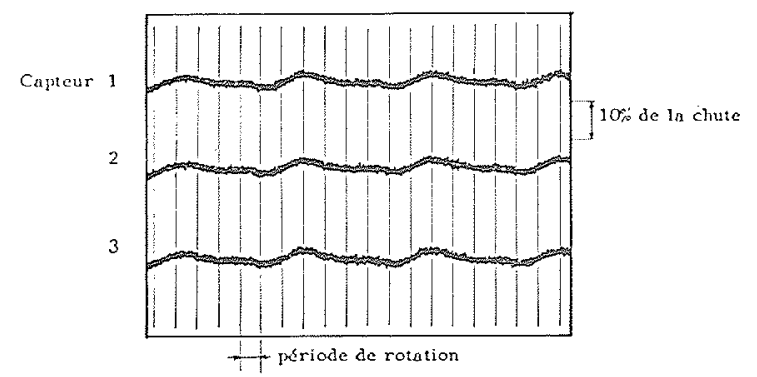

Figure 4. - Pulsation de la pression dans la section C (Les trois signaux sont en phase entre eux)

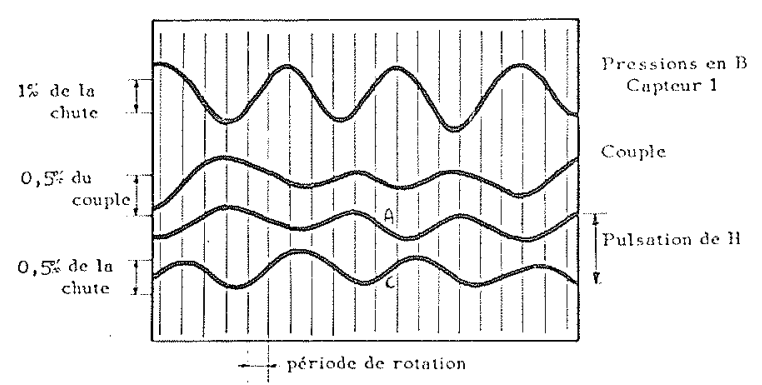

Figure 5. - Pulsations de la pression sous la roue, du couple et de la chute (Signaux filtrés) 


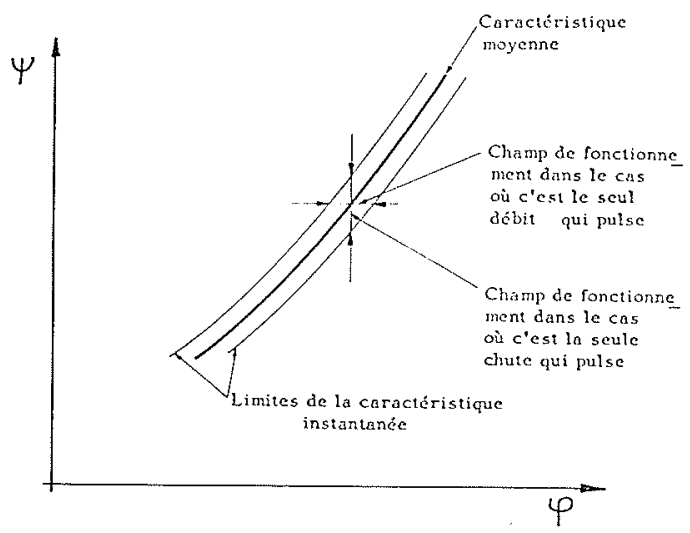

Figure 6. - Pulsation de la caractéristique de la turbine à charge partielle (Ouverture constante du distributeur)

c) La pulsation dans la section A est en opposition de phase par rapport à celle dans la section $\mathrm{C}$ (fig. 5 ).

En conclusion, on peut dire que la caractéristique chute/ débit pulse de la façon indiquée schématiquement sur la figure 6. Les conséquences les plus évidentes de ce phénomène sont : les pulsations de pression qui se propagent le long des conduites amont et aval, et les pulsations de puissance et de couple indiquées sur la figure 5 .

A la limite (fig. 6), la pulsation de la caractéristique peut entraîner les pulsations du débit ou de la seule chute : en effet le phénomène est plus complexe et le point de fonctionnement se déplace sur une ellipse qui est fonction des caractéristiques du système hydraulique.
Des essais sur modèle peuvent fournir des observations intéressantes comme le montrent les enregistrements projetés. Dans nos laboratoires nous sommes en train de perfectionner les systèmes de détection instantanée des différentes grandeurs (chute, couple, charge et vitesse de rotation) dans le but d'une recherche systématique sur cet important problème.

M. SCHMITT. - Je signale l'existence d'un débitmètre à ultrasons qui fonctionne très bien et dont la bande passante est fonction du diamètre de la canalisation et de la vitesse du son Pour un diamètre de $150 \mathrm{~mm}$ et de l'eau, elle est supérieure à $100 \mathrm{~Hz}$.

M. BERHAULT. - La mesure directe de limpédance complexe locale d'un dispositif (pompe, vannes, ....) est actuellement réalisée, avec succès, en utilisant une combinaison film chaud (DISA) capteur piézoélectrique de pression. C'est, par exemple, le cas de l'équipe du Professeur BOWS à l'Université de Bath en Grande Bretagne.

Mme JOLAS. - Il faut également citer les travaux de $\mathrm{M}$. BRENNEN (California Institute of Technology) sur la réponse dynamique d'une pompe axiale en régime cavitant et non cavitant $\left({ }^{l}\right)$ et rappeler à ce sujet, que lors des essais, la mesure des fluctuations de vitesse a été effectuée par vélocimétrie laser à effet Doppler.

M. CHEVALIER. - S'il n'y a plus d'autres questions, il me reste à remercier les auditeurs et à vous remercier tous d'avoir participé ă la discussion et d'être venus nombreux assister à cette séance.

La Séance est levée à $12 \mathrm{H} 15$.

(1). The unsteady dynamic characterization of hydraulic systems with emphasis on cavitation and turbomachines. Vol. 1 Proc. of Joint ASCE/IAHR/ASME Symp. on Design and Operation of Fluid Machinery, Fort Collins (Colo.) June 1978 pp. 97-107. 